\title{
RESEARCH ON ELEMENTARY PARTICLE PHYSICS
}

Task P-Studies in Theory and Phenomenology of Elementary Particles

\author{
Annual Progress Report \\ for Period November 1, 1992-October 31, 1993 \\ Co-Principal Investigators: L. E. Holloway, T. A. O'Halloran \\ Task Director: L. M. Jones
}

University of nlinois at Urbana-Champaign

Department of Physics

Loomis Laboratory of Physics

1110 West Green Street

Urbana, Illinois

May 1993

Prepared for

THE U.S. DEPARTMENT OF ENERGY 


\begin{abstract}
$\underline{\text { Abstract }}$
Continued progress has been made in isolating the variables needed to completely specify a quark or gluon jet, independent of the process in which it is created. We have evidence of a slow, but statistically significant, dependence on the sub-energy of the hard process in which the jet arises. Once this is taken into account, jets from $e^{+} e^{-}$are consistent with those created in $p \bar{p}$. Neural network jet identification studies will be based on this expanded set of variables.

Work on the new type of Dirac equation for a relativistic oscillator was presented at the International Conference on High Energy Physics in Dallas, and will be published in the Proceedings of the Conference. Theoretical studies involving jet signals for theoretically predicted (but as yet undiscovered) particles are underway.
\end{abstract}

The chief people working on this Task are Lorella Jones (Task leader), and graduate students Mary Ann Graham and Rafal Filipczyk.

\title{
DISCLAIMER
}

This report was prepared as an account of work sponsored by an agency of the United States Government. Neither the United States Government nor any agency thereof, nor any of their employees, makes any warranty, express or implied, or assumes any legal liability or responsibility for the accuracy, completeness, or usefulness of any information, apparatus, product, or process disclosed, or represents that its use would not infringe privately owned rights. Reference herein to any specific commercial product, process, or service by trade name, trademark, manufacturer, or otherwise does not necessarily constitute or imply its endorsement, recommendation, or favoring by the United States Government or any agency thereof. The views and opinions of authors expressed herein do not necessarily state or reflect those of the United States Government or any agency thereof. 


\section{Phenomenology}

It has been our long range goal to devise techniques based on a)isolation of crucial jet variables, and b)neural network calculations involving these jet variables, to identify the originating parton for Quantum Chromodynamic jets, on a one-toone basis. To this end, over the past several years, we have developed an analysis method using Lorentz-invariant properties of individual jets and demonstrated that quarks and gluons have different statistical properties in these variables ${ }^{1}$. Also, we have demonstrated ${ }^{2}$ using this method that detailed differences exist between the predictions of the two most commonly used QCD packages, even after they have been fit to more global properties of $e^{+} e^{-}$data; and that this approach could thus be used to distinguish whether either of them provides a really accurate representation of experiment.

Over the last year, large numbers of 2-jet and 3-jet events in $e^{+} e^{-}$have been generated at 2 different energies using the Herwig and Jetset (Lund) Monte Carlo packages, and a similar number of 2-jet events have been generated in $p \bar{p}$ collisions (at Tevatron energies) using Herwig and Pythia (Lund). We have studied these jets, using our previous method of analysis. At present this study is partially completed, with the following (preliminary) results:

i)For the case of jets generated using the Herwig package, the jet variables previously used in our classification scheme (the longitudinal momentum fractions of all the hard particles in the jet, handled according to the correlation bins summarized in Table I, and the jet mass) need to be augmented by the hard process (center-of-mass) subenergy of the reaction in which the jet originated. The longitudinal momentum distribution of the hadrons in the jets has a smaller dependence on this variable than it does on the jet mass, but it appears to be statistically important.

Figure 1 shows correlation bin frequencies for quark jets in the $e^{+} e^{-} \rightarrow 2 j$ et process for three different center-of-mass energies : 36,60 , and $90 \mathrm{GeV}$. We 
see that the plots of correlation bin frequencies are quite similar within a given mass range, for the different energies, showing that the general approach is valid. However, when the Kolmogorov-Smirnov statistic is calculated comparing data from any two of these energies, it turns out that they are quite unlikely to be the same. These values are presented in Tables II,III and IV. As explained in Ref. 2, K-S statistics larger than 2 may be taken as indicating that the Monte Carlo events were generated from different distributions.

Although the conclusions are less clear because at present we lack statistics in the $p \bar{p}$ sample at large hard sub-process energies, similar results are obtained for jets produced in hadronic reactions. In Figure 2 and Table $\mathrm{V}$ we present data for the case of jets generated in $p \bar{p}$ reactions at Tevatron energies. Once again the histograms showing correlation bin frequencies for a given jet mass range are rather similar when one compares the two center-of-mass energy ranges $\mathrm{A}\left(1100<\hat{s}<1600 \mathrm{GeV}^{2}\right)$ and $\mathrm{B}\left(2500<\hat{s}<4900 \mathrm{GeV}^{2}\right)$. This is true for both quark and gluon jets (Figs $2 a$ and $2 b$ ). In this case the K-S statistic comparing the two energies shows less difference (ste Table V); but a much larger data sample needs to be generated to be sure whether or not there is an energy dependence here.

ii) Once the dependence mentioned in (i) is taken into account, the jets generated by Herwig in $e^{+} e^{-}$reactions are, on a statistical basis, the same as those generated by Herwig for $p \bar{p}$ reactions. Data demonstrating this is presented in Figures $3 \mathrm{a}$ and $3 \mathrm{~b}$ (for $e^{+} e^{-}$data at 36 and $60 \mathrm{GeV}$ and comparable $p \bar{p}$ subenergy slices), and the comparable K-S statistics are shown in Table VI.

We believe that this effect should also be taken into account in comparing jets from $e^{+} e^{-} \rightarrow 3 j$ et data with jets from hadronic reactions. A great deal of preliminary work suggests that use should be made of the appropriate 
hard process subenergy in the three jet process. Further studies along these lines are in progress.

iii)Analysis of events generated using the Lund packages is ongoing. It has been compticated somewhat by the fact that there are two packages (Pythia and Jetset) and it has taken a while to completely understand both of them. Events have been generated using each package and they are being analyzed using the methods used for the Herwig case.

We are now returning to our previous neural network studies. This should "complete the loop" and finish the overall project by determining the theoretical "best" one-by-one identification of the jets.

We hope to complete this project within the next year, to coincide with the graduation of Mary Ann Graham, the senior student involved in the project.

\section{Theory}

Work on the new type of Dirac equation for a relativistic oscillator was presented at the International Conference on High Energy Physics in Dallas, and will be published in the Proceedings of the Conference ${ }^{3}$.

Rafal Filipczyk, a graduate student, has spent the last year studying the mathematics and physics of grand unified models of various sorts. (This material is only partially contained in the particle physics courses taught here presently). Most recently, he has been calculating the expected sizes of signals for "exotic" particles such as top quarks and Higgs particles in former and present $p \bar{p}$ reactions. This is serving both as a training exercise and as a preparation to finding some larger calculations for a thesis topic. 


\section{Comments}

There have been a number of students involved in the jet calculations during the last year. Mary Ann Graham, whose thesis involves this work, has been involved continuously. Chen $\mathrm{Ji}$, an undergraduate student, worked on the generation of jets in $p \bar{p}$ using Pythia; -during the Fall of 1992. Johann Beda, a first year graduate student, worked on the generation of jets in $p \bar{p}$ using Herwig, during the Summer of 1992. Inclusion of the additional students has accelerated parts of the work, while providing them with additional training. However, there has also been some time lost in the education of the additional temporary workers.

Much of the work has been done using the facilities of the National Center for Supercomputing Applications at Urbana. We would have difficulties storing and manipulating all the generated data without these facilities, but there have been considerable delays introduced into some of the work by this arrangement. The detailed listing of these problems is not so important as the following conclusion: it is more important, at national computing facilities, to have STABLE equipment configurations (so that both consultants and users can cope) than it is to have the very latest equipment.

We have obtained, with the funds for this year, a Vxt $2000 \mathrm{X}$-window terminal. This equipment is housed in Dr. Jones's office, and used by all members of the group.

From August 13, 1992 to June 30, 1994, Prof. Jones is serving half-time as Interim Director of the Computer-based Education Research Lab on the UIUC campus. This is a natural extension of her long-time interest in computer- based physics education, which uses the facilities of this lab. It takes the place of teaching in the Physics department. The main impact of this appointment on the work being performed under this grant is that it reduces Prof. Jones's DOE summer appointment for 1993 from two months to one month. This should "free up" money for additional student salaries. It will also, however, reduce the actual contribution of Dr. Jones to the work. 
References

1)L.M.Jones, Towards a Systematic Jet Ciassification. Phys. Rev. D $\underline{42}$, p.811 (1990)

2)M. A. Graham, L.M. Jones and P. Daumerie, Comparison of Jet Fragmentation Schemes. Phys. Rev. D年, p.222 (1992)

3)L.M. Jones, Another Dirac Oscillator, Proceedings of the XXVI International Conference on High Energy Physics, to be published. 
TABLE I. Definition of the Correlation Bins.

\begin{tabular}{|c|c|c|c|c|c|c|c|}
\hline bin & & bin & & bin & & bin & \\
\hline 1 & 9 & 26 & 5 & 51 & 3,3 & 76 & $2,2,1,1,1$ \\
\hline 2 & $8,1 \cdots \cdots$ & 27 & $4,4,1$ & 52 & $3,2,2,2$ & 77 & $2,2,1,1$ \\
\hline 3 & 8 & 28 & 4,4 & 53 & $3,2,2,1,1$ & 78 & $2,2,1$ \\
\hline 4 & 7,2 & 29 & $4,3,2$ & 54 & $3,2,2,1$ & 79 & 2,2 \\
\hline 5 & $7,1,1$ & 30 & 4,$3 ; 1,1$ & 55 & $3,2,2$ & $80^{\circ}$ & $2,1,1,1,1,1,1,1$ \\
\hline 6 & 7,1 & 31 & $4,3,1$ & 56 & $3,2,1,1,1,1$ & 81 & $2,1,1,1,1,1,1$ \\
\hline 7 & 7 & 32 & 4,3 & 57 & $3,2,1,1,1$ & 82 & $2 ; 1,1,1,1,1$ \\
\hline 8 & 6,3 & 33 & $4,2,2,1$ & 58 & $3,2,1,1$ & 83 & $2,1,1,1,1$ \\
\hline 9 & $6,2,1$ & 34 & $4,2,2$ & 59 & $3,2,1$ & 84 & $2,1,1,1$ \\
\hline 10 & 6,2 & 35 & $4,2,1,1,1$ & 60 & 3,2 & 85 & $2,1,1$ \\
\hline 11 & $6,1,1,1$ & 36 & $4,2,1,1$ & 61 & $3,1,1,1,1,1,1$ & 86 & 2,1 \\
\hline 12 & $6,1,1$ & 37 & $4,2,1$ & 62 & $3,1,1,1,1,1$ & 87 & 2 \\
\hline 13 & 6,1 & 38 & 4,2 & 63 & $3,1,1,1,1$ & 88 & $1,1,1,1,1,1,1,1,1$ \\
\hline 14 & 6 & 39 & $4,1,1,1,1,1$ & 64 & $3,1,1,1$ & 89 & $1,1,1,1,1,1,1,1$ \\
\hline 15 & 5,4 & 40 & $4,1,1,1,1$ & 65 & $3,1,1$ & 90 & $1,1,1,1,1,1,1$ \\
\hline 16 & $5,3,1$ & 41 & $4,1,1,1$ & 66 & 3,1 & 91 & $1,1,1,1,1,1$ \\
\hline 17 & 5,3 & 42 & $4,1,1$ & 67 & 3 & 92 & $1,1,1,1,1$ \\
\hline 18 & $5,2,2$ & 43 & 4,1 & 68 & $2,2,2,2,1$ & 93 & $1,1,1,1$ \\
\hline 19 & $5,2,1,1$ & 44 & 4 & 69 & $2,2,2,2$ & 94 & $1,1,1$ \\
\hline 20 & $5,2,1$ & 45 & $3,3,3$ & 70 & $2,2,2,1,1,1$ & 95 & 1,1 \\
\hline 21 & 5,2 & 46 & $3,3,2,1$ & 71 & $2,2,2,1,1$ & 96 & 1 \\
\hline 22 & $5,1,1,1,1$ & 47 & $3,3,2$ & 72 & $2,2,2,1$ & 97 & no hard particles \\
\hline 23 & $5,1,1,1$ & 48 & $3,3,1,1,1$ & 73 & $2,2,2$ & & \\
\hline 24 & $5,1,1$ & 49 & $3,3,1,1$ & 74 & $2,2,1,1,1,1,1$ & & \\
\hline 25 & 5,1 & 50 & $3,3,1$ & 75 & $2,2,1,1,1,1$ & & \\
\hline
\end{tabular}

TABLE II. Values of the K-S Statistic for comparison of quark jets created in $e^{+} e^{-} \rightarrow 2$ jets at center of mass energy $36 \mathrm{GeV}$ with those created at $60 \mathrm{GeV}$.

\begin{tabular}{lrrr}
\hline \hline Jet Mass & $D_{M N}$ & $\mathrm{M}^{\mathbf{2}}$ & $\mathrm{N}^{\mathrm{b}}$ \\
\hline $0-4 \mathrm{GeV}$ & 1.37 & 2281 & 1864 \\
$4-8 \mathrm{GeV}$ & 5.45 & 2559 & 8733 \\
$8-12 \mathrm{GeV}$ & .63 & 22 & 2309 \\
\hline \hline
\end{tabular}

${ }^{2} \mathrm{M}$ is the number of jets in the $36 \mathrm{GeV}$ histogram.

${ }^{b} \mathrm{~N}$ is the number of jets in the $60 \mathrm{GeV}$ histogram.

TABLE III. Values of the K-S Statiatic for comparieon of quark jets created in $e^{+} e^{-} \rightarrow 2$ jets at center of mass energy $60 \mathrm{GeV}$ with those created at $90 \mathrm{GeV}$.

\begin{tabular}{l|rlr}
\hline \hline Jet Mass & $D_{M N}$ & $M^{2}$ & $N^{b}$ \\
\hline $0-4 \mathrm{GeV}$ & .31 & 1864 & 593 \\
$4-8 \mathrm{GeV}$ & 3.68 & 8733 & 5986 \\
i-12 GeV & 7.53 & 2309 & 4518 \\
\hline \hline
\end{tabular}

${ }^{2} \mathrm{M}$ is the number of jets in the $60 \mathrm{GeV}$ histogram.

${ }^{b} \mathrm{~N}$ is the number of jets in the $90 \mathrm{GeV}$ histogram. 
TABLE IV. Values of the K-S Statistic for comparison of quark jets created in $e^{+} e^{-} \rightarrow 2 j e t s$ at center of mass energy $36 \mathrm{GeV}$ with those created at $90 \mathrm{GeV}$.

\begin{tabular}{lrrr}
\hline \hline Jet Mass & $D_{M N}$ & $M^{\mathrm{a}}$ & $\mathrm{N}^{\mathrm{b}}$ \\
\hline $0-4 \mathrm{GeV}$ & 1.06 & 2281 & 593 \\
$4-8 \mathrm{GeV}$ & 7.64 & 2559 & 5986 \\
$8-12 \mathrm{GeV}$ & 1.30 & 22 & 4518 \\
\hline \hline
\end{tabular}

' $\mathrm{M}$ is the number of jets in the $36 \mathrm{GeV}$ histogram.

${ }^{b} \mathrm{~N}$ is the number of jets in the $90 \mathrm{GeV}$ histogram.

TABLE V. Values of the K-S Statistic for comparison of jets from $p \bar{p} \rightarrow 2$ jets at two different hard process sub-energies. The two samples represent sub process center of mass energy squared of $1100 \mathrm{GeV}^{2} \leq \hat{S} \leq 1600 \mathrm{GeV}^{2}$ and $2500 \mathrm{GeV}^{2} \leq \hat{S} \leq 4900 \mathrm{GeV}^{2}$.

\begin{tabular}{lrrrrrr}
\hline & & Gluon Jets & \multicolumn{3}{c}{ Quark Jets } \\
Jet Mass & $D_{M N}$ & \multicolumn{1}{c}{$\mathrm{M}^{2}$} & \multicolumn{1}{c}{$\mathrm{N}^{\mathrm{b}}$} & $D_{M N}$ & \multicolumn{1}{c}{$\mathrm{M}^{2}$} & $\mathrm{~N}^{\mathrm{b}}$ \\
\hline $0-4 \mathrm{GeV}$ & .75 & 933 & 19 & .58 & 471 & 39 \\
$4-8 \mathrm{GeV}$ & .60 & 4551 & 528 & .76 & 1096 & 217 \\
$8-12 \mathrm{GeV}$ & 2.14 & 654 & 546 & 1.12 & 117 & 131 \\
\hline \hline
\end{tabular}

${ }^{2} \mathrm{M}$ is the number of jets in the $1100 \mathrm{GeV}^{2} \leq S \leq 1600 \mathrm{GeV}^{2}$ histogram.

${ }^{b} \mathrm{~N}$ is the number of jets in the $2500 G e V^{2} \leq \hat{S} \leq 4900 G e V^{2}$ histogram.

TABLE VI. Values of the K-S Statistic for comparison between quark jets from $e^{+} e^{-} \rightarrow 2 j e t s$ and $p \bar{p} \rightarrow 2$ jets. The 36 and $60 \mathrm{GeV}$ refer to the center of mass energy of the $e^{+} e^{-}$events. $\hat{S}$ is the center of mass energy squared of the hard subprocess in $p \bar{p}$.

\begin{tabular}{|c|c|c|c|c|}
\hline Jet Mass & $\hat{\hat{S}}$ & $D_{M N}$ & $\mathbf{M}^{\mathbf{A}}$ & $\mathrm{N}^{b}$ \\
\hline & & \multicolumn{3}{|c|}{$36 \mathrm{GeV} e^{+} e^{-}$events } \\
\hline $0-4 \mathrm{GeV}$ & allt & 1.84 & 2281 & 6000 \\
\hline $4-8 \mathrm{GeV}$ & all & 1.61 & 2559 & 6252 \\
\hline $8-12 \mathrm{GeV}$ & all & .65 & 22 & 578 \\
\hline $0.4 \mathrm{GeV}$ & $1100-1600$ & 1.29 & 2281 & 471 \\
\hline $4-8 \mathrm{GeV}$ & $1100-1600$ & 1.34 & 2559 & 1096 \\
\hline \multirow[t]{2}{*}{$8-12 \mathrm{GeV}$} & $1100-1600$ & .62 & 22 & 117 \\
\hline & & \multicolumn{3}{|c|}{$60 \mathrm{GeV} \mathrm{e}^{+} e^{-}$events } \\
\hline $0-4 \mathrm{GeV}$ & all & 2.93 & 1864 & 6000 \\
\hline $4-8 \mathrm{GeV}$ & all & 7.59 & 8733 & 6252 \\
\hline 8-12 GeV & all & 1.17 & 2309 & 578 \\
\hline $0-4 \mathrm{GeV}$ & $2500-4900$ & .73 & 1864 & 39 \\
\hline $48 \mathrm{GeV}$ & $2500-4900$ & 1.24 & 8733 & 217 \\
\hline 8-12 GeV & $2500-4900$ & 1.33 & 2309 & 131 \\
\hline
\end{tabular}

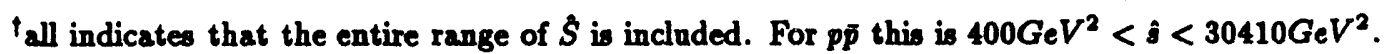

${ }^{-} M$ is the number of jets in the $e^{+} e^{-}$histogram.

${ }^{b} \mathrm{~N}$ is the number of jets in the $p \bar{p}$ histogram. 
Figure 1. Plots of correlation bin frequencies for quark jets from $e^{+} e^{-} \rightarrow 2 j e t s$. The plots are divided according to jet masses (0-4 GeV , 4-8 GeV and 8-12 GeV) and center of mass energy ( $36 \mathrm{GeV}, 60 \mathrm{GeV}$ and $90 \mathrm{GeV})$.

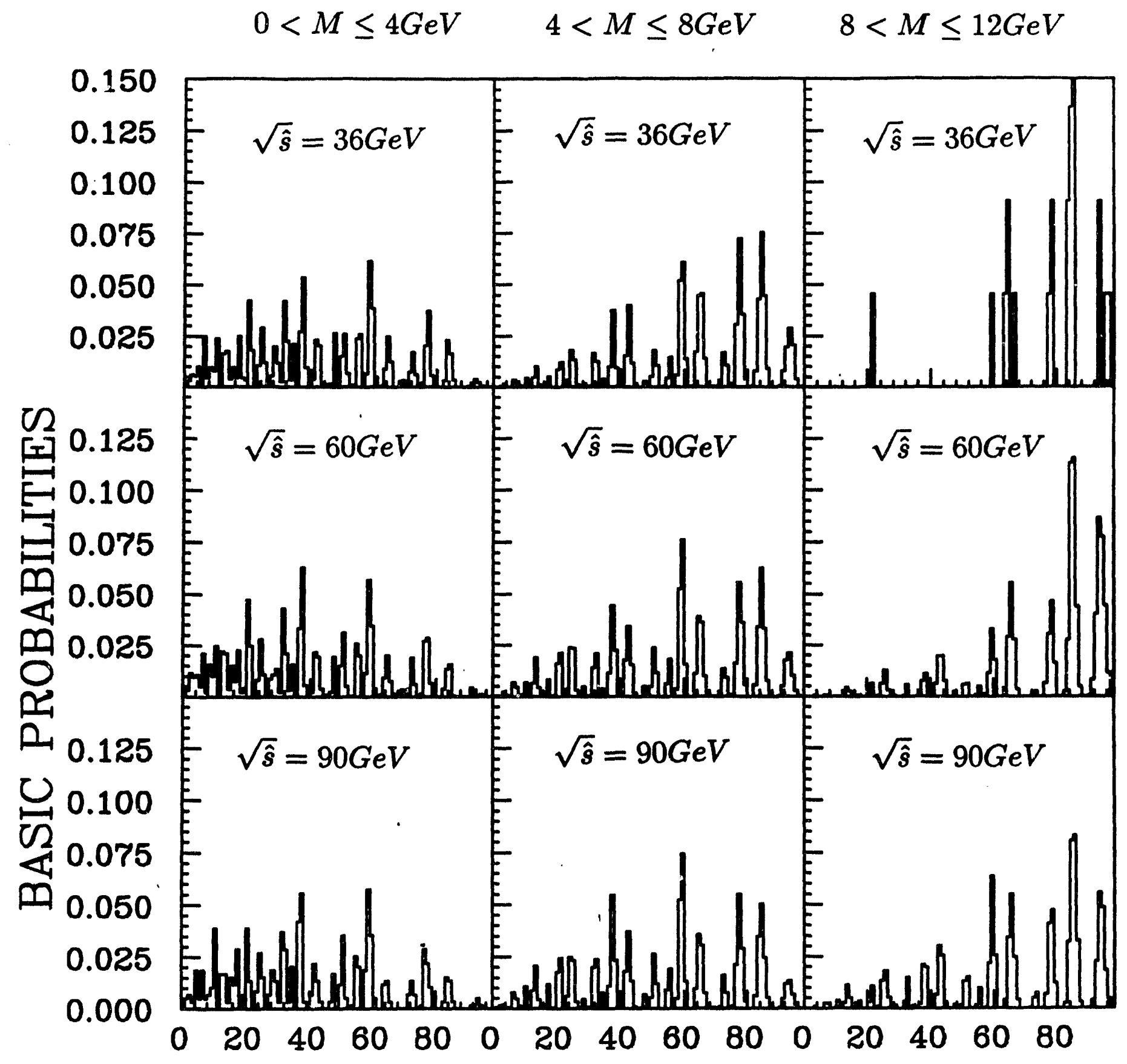

BIN 
Figure 2. Plots of correlation bin frequencies for jets from $p \ddot{p} \rightarrow 2 j e t s$. The plots are divided according to jet masses (0-4 GeV , 4-8 GeV and 8-12 GeV) and center of mass energy squared of the 2 jet system (1100-1600 $\mathrm{GeV}^{2}$ and 2500-4900 $\mathrm{GeV}^{2}$ ).

(a) Light quark jets.

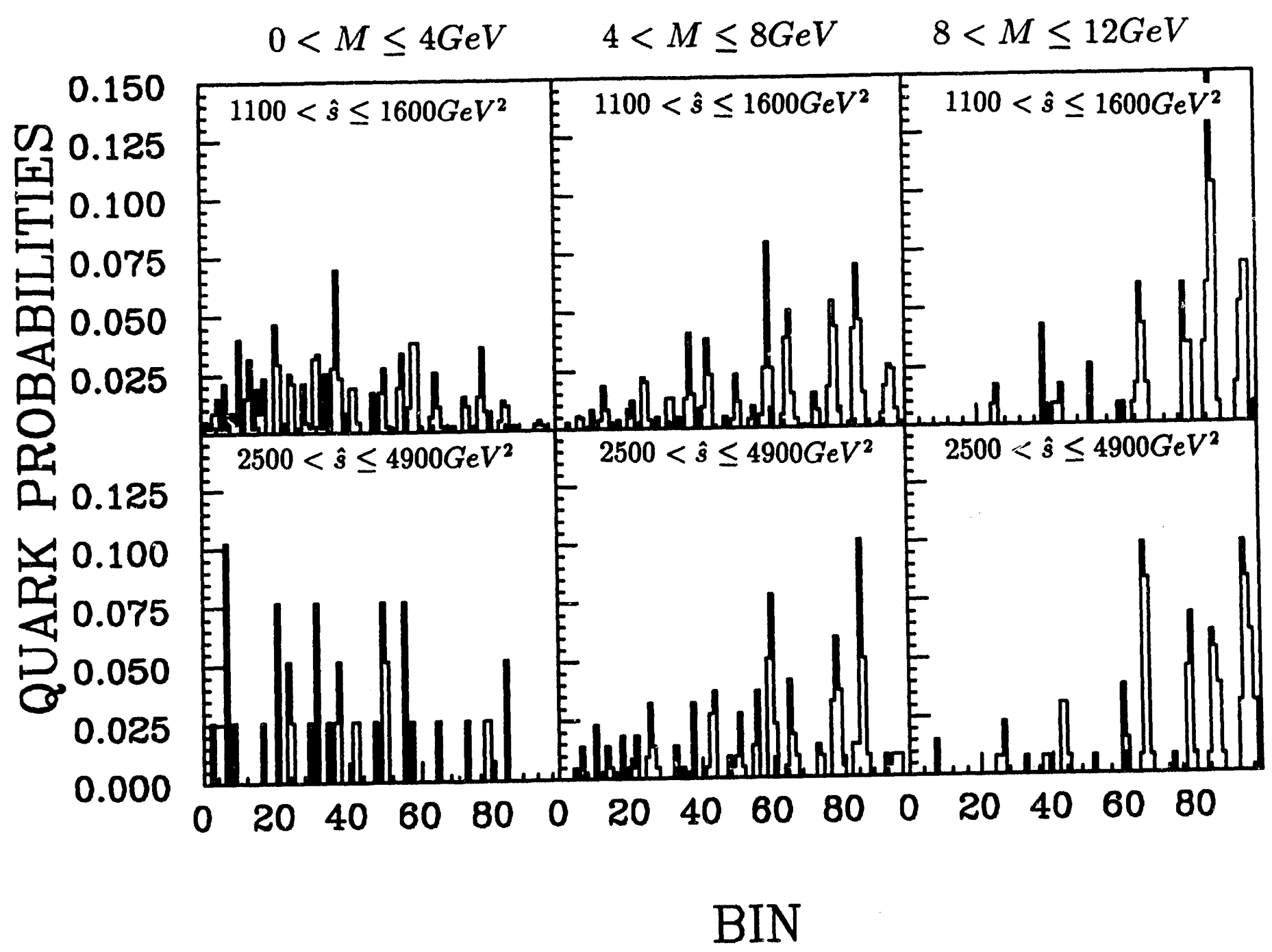


Figure 2. (b) Gluon jets.

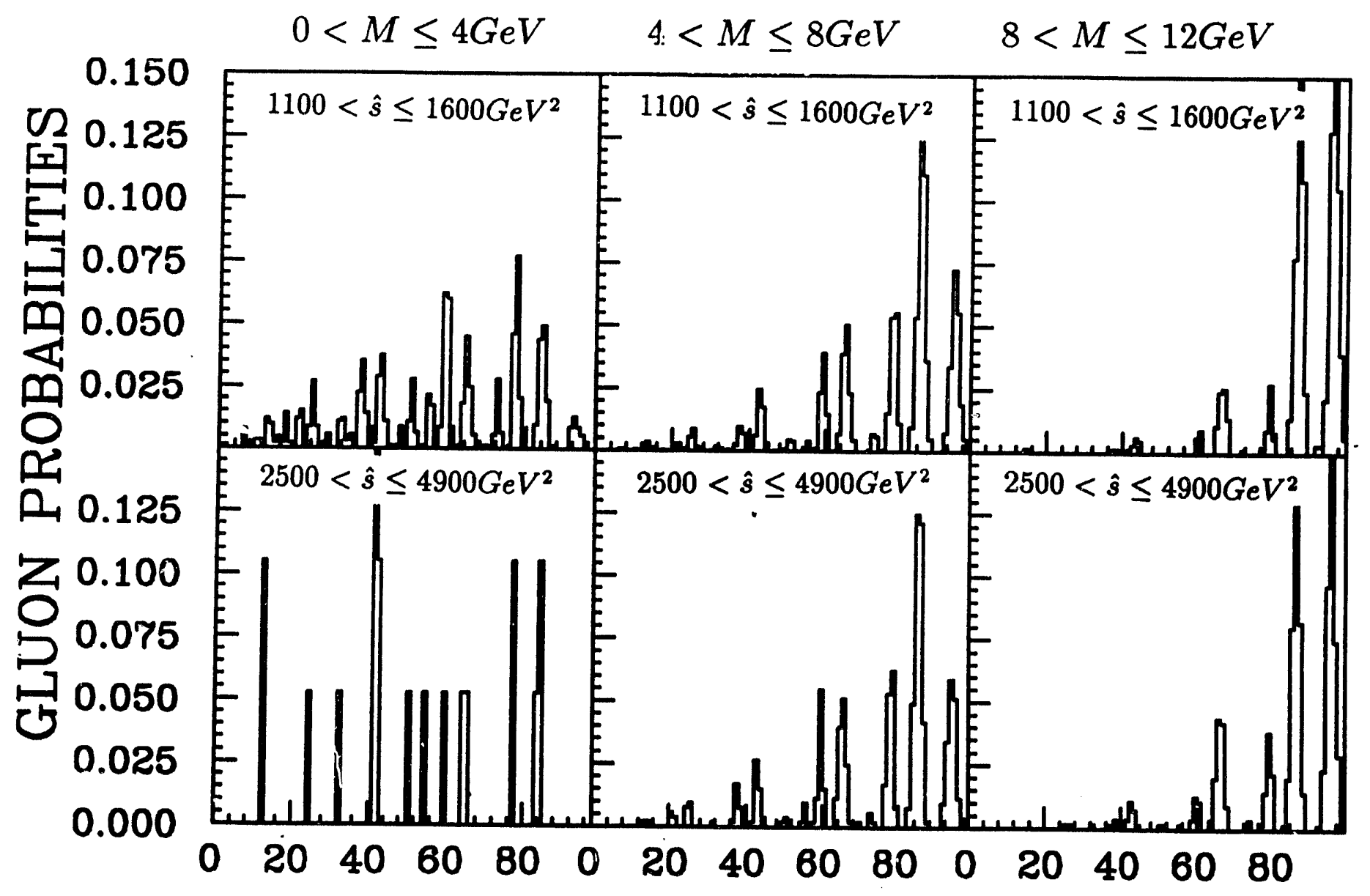

BIN 
Figure 3. Plots of correlation bin frequencies for jets from $e^{+} e^{-} \rightarrow 2 j$ ets and $p \bar{p} \rightarrow$ $2 j e t s$. The plots are divided into jet masses $(0-4 \mathrm{GeV}, 4-8 \mathrm{GeV}$ and 8-12 GeV).

(a) The $p \bar{p}$ events have center of mass energy squared $1100-1600 \mathrm{GeV}^{2}$ for the hard subprocess, and the $e^{+} e^{-}$are at center of mass energy $36 \mathrm{GeV}$.

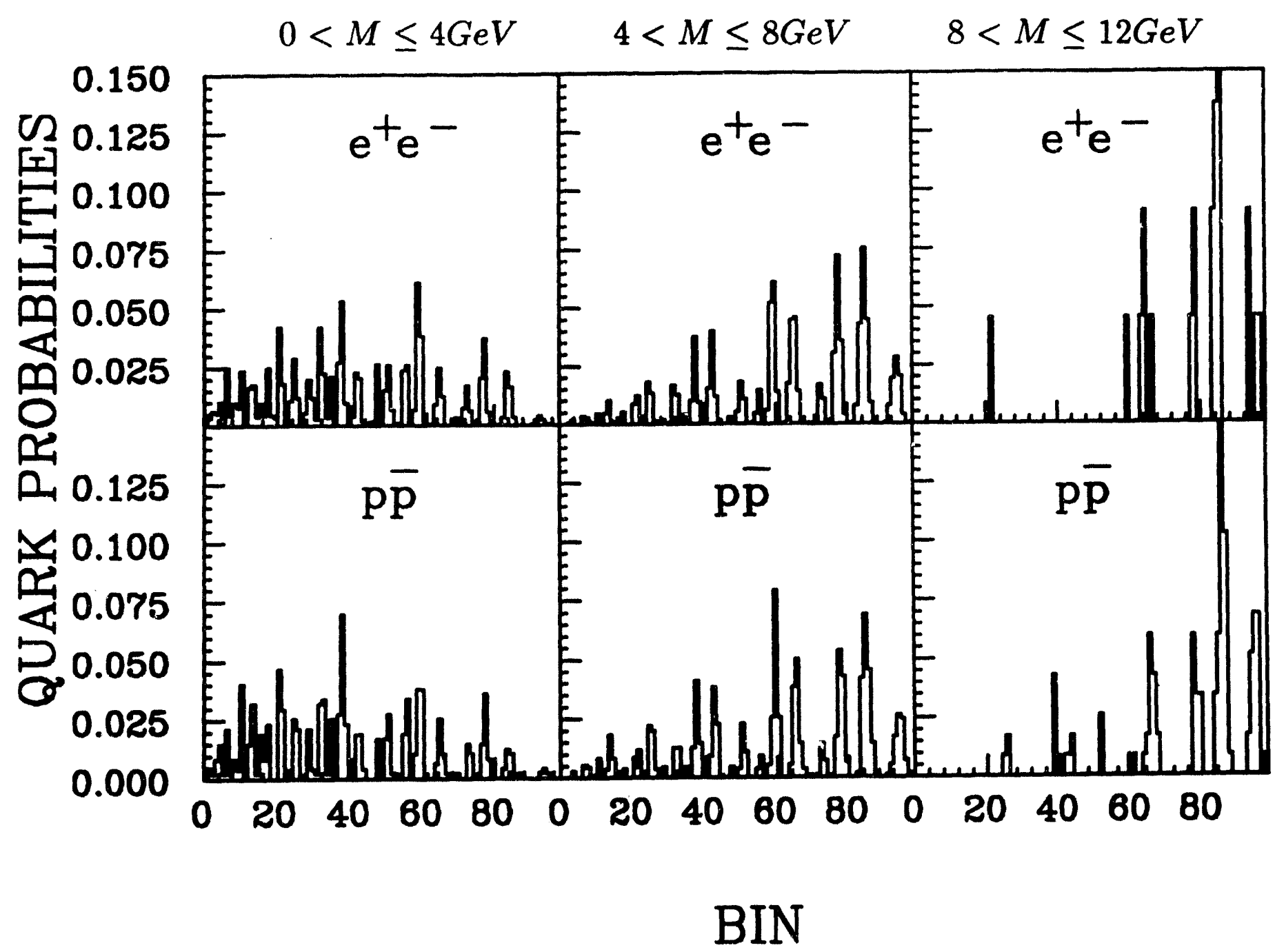


Figure 3. (b) $p \bar{p}$ events with hard sub process center of mass energy squared of 2500$4900 \mathrm{GeV}^{2}$ compared with $e^{+} e^{-}$events at $60 \mathrm{GeV}$.

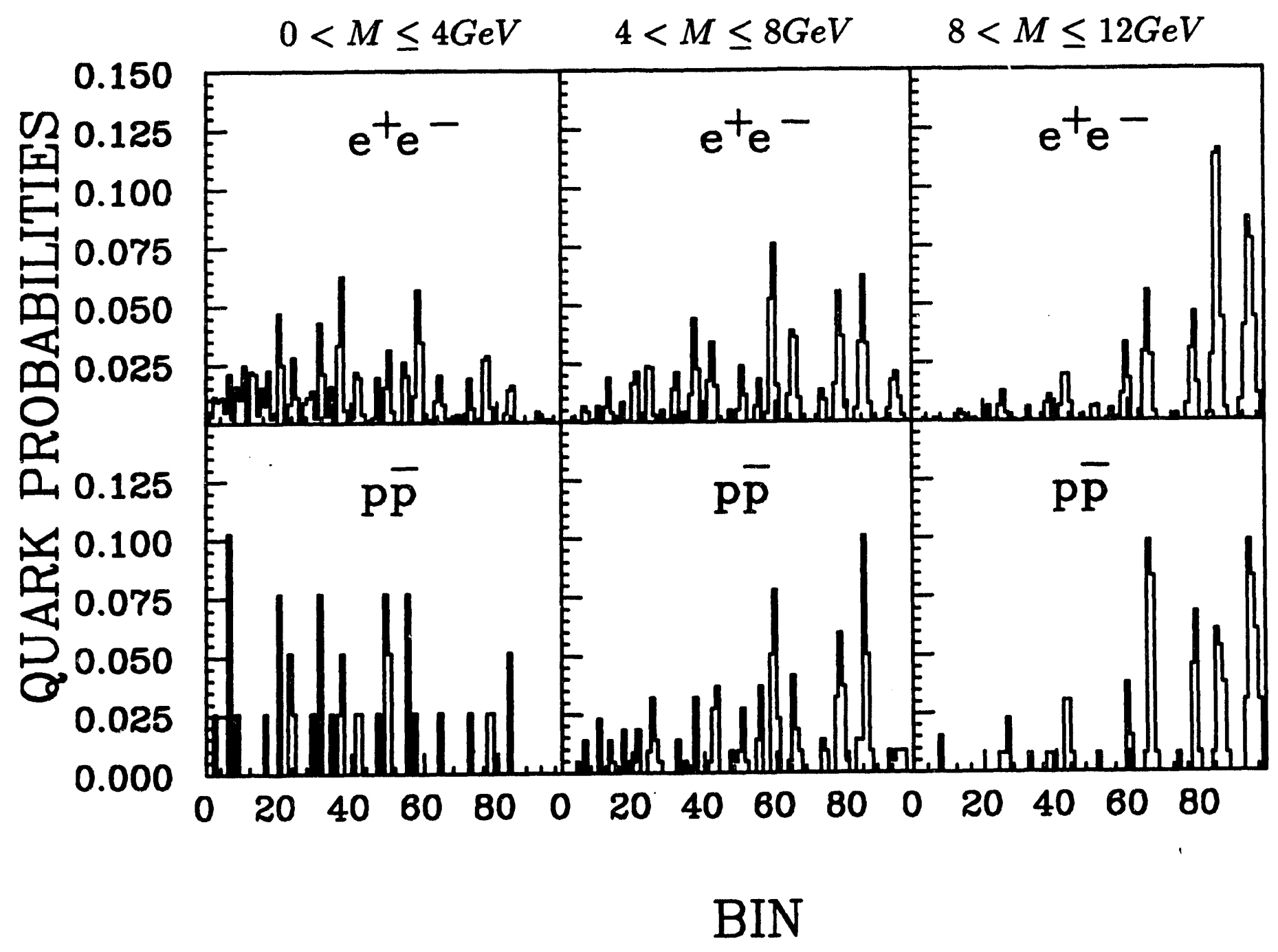


b
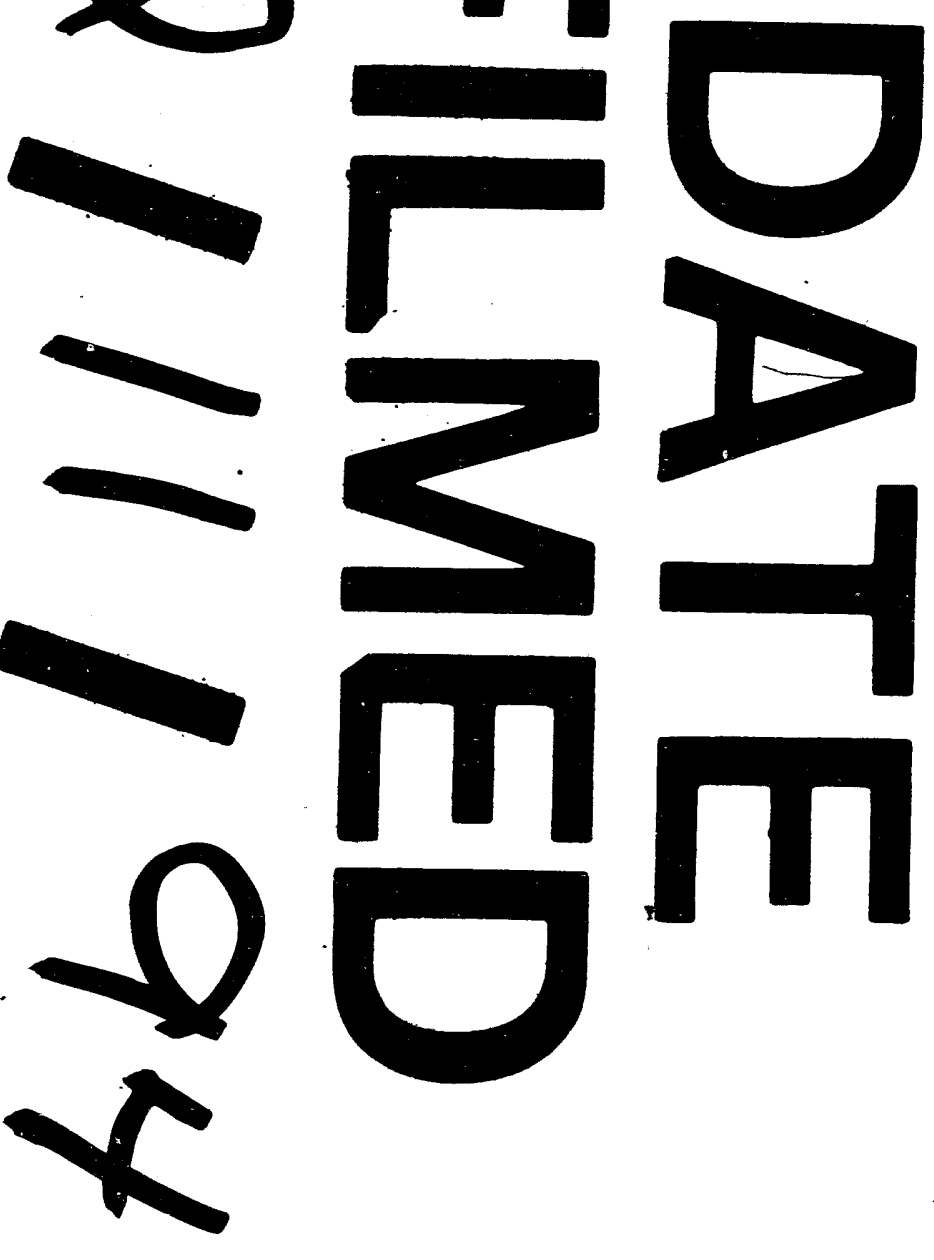

$=$

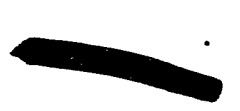

$\checkmark$

$20 \pi$

$x$
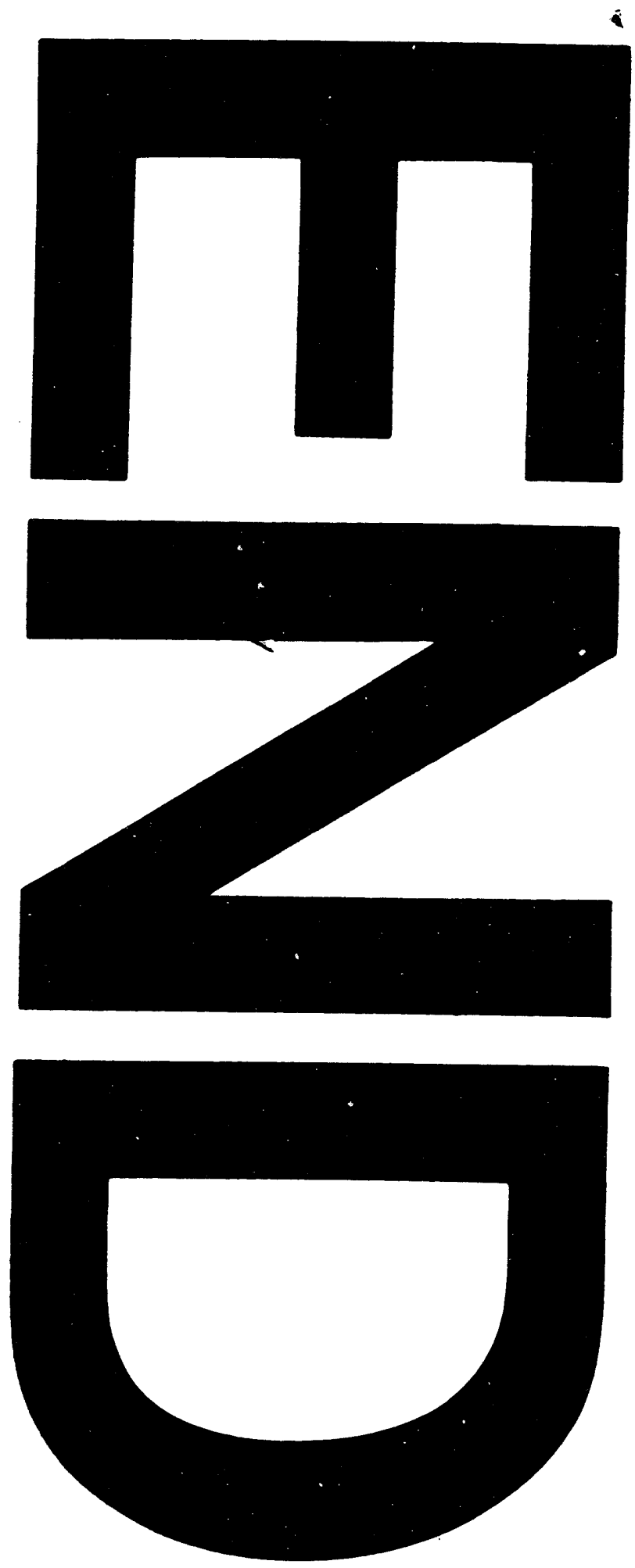
